\title{
Unstable Nonlocal Interface Dynamics
}

\author{
Matteo Nicoli, ${ }^{1}$ Rodolfo Cuerno, ${ }^{1}$ and Mario Castro ${ }^{2}$ \\ ${ }^{1}$ Departamento de Matemáticas and Grupo Interdisciplinar de Sistemas Complejos (GISC), Universidad Carlos III de Madrid, \\ Avenida de la Universidad 30, E 28911 Leganés, Spain \\ ${ }^{2}$ GISC and Grupo de Dinámica No Lineal (DNL), Escuela Técnica Superior de Ingeniería (ICAI), Universidad Pontificia Comillas, \\ E 28015 Madrid, Spain
}

\begin{abstract}
Nonlocal effects occur in many nonequilibrium interfaces, due to diverse physical mechanisms like diffusive, ballistic, or anomalous transport, with examples from flame fronts to thin films. While dimen sional analysis describes stable nonlocal interfaces, we show the morphologically unstable condition to be nontrivial. This is the case for a family of stochastic equations of experimental relevance, paradigmatically including the Michelson Sivashinsky system. For a whole parameter range, the asymptotic dynamics is scale invariant with dimension independent exponents reflecting a hidden Galilean symmetry. The usual Kardar Parisi Zhang nonlinearity, albeit irrelevant in that parameter range, plays a key role in this behavior.
\end{abstract}

DOI: 10.1103/PhysRevLett.102.256102

PACS numbers: 68.35.Ct, 05.45. a, 47.54. r

The interface dynamics of many nonequilibrium systems arises from the interplay between nonlocal interactions and morphological instabilities. Examples range from flame front propagation to thin film growth [1]. Often, although the basic physical interactions are short-ranged and local, the evolution is driven by nonlocal effects implicitly (via projection of the overall dynamics on the interface) or explicitly (as in elastic media or viscous flow) [1]. These nonlocalities appear in many fields, diffusion-limited growth being a prominent example. Here, although diffusion events of aggregating units are local, the morphology of a growing cluster is dominated by shadowing of the most prominent surface features over less exposed ones. Hence, the local growth velocity depends on the global surface shape. This moreover leads to the classic Mullins-Sekerka (MS) morphological instability [1], whereby prominent features grow faster. Still, nonlocality and morphological stability are independent properties. Thus, we may consider diffusion-limited erosion (DLE) [2,3], qualitatively relevant to experiments on, e.g., ion irradiation smoothing [4]. Here the most exposed surface features are eroded faster, leading to nonlocal stable interface evolution in which differences in surface height are smoothed out.

Often [3], the nonequilibrium dynamics of these interfaces can be cast into a stochastic equation for the surface height $h(\mathbf{r}, t)$ at time $t$ and point $\mathbf{r}$ on a $d$-dimensional substrate. Assuming translational and rotational symmetry, we propose the following equation (after space Fourier transform $\mathcal{F}$ )

$$
\begin{aligned}
\partial_{t} h_{\mathbf{k}}(t)= & \left(-\nu k^{\mu}-\mathcal{K} k^{m}-\mathcal{N} k^{n}\right) h_{\mathbf{k}}(t)+\frac{\lambda}{2} \mathcal{F}\left[(\nabla h)^{2}\right] \\
& +\eta_{\mathbf{k}}(t) .
\end{aligned}
$$

Here, $\mu, m, n, \mathcal{K}$, and $\mathcal{N}$ are positive constants $(0<\mu \leq$ $2, m \geq 2$, and $n>m$; see below), the linear dispersion relation $\omega_{\mathbf{k}} \equiv-\nu k^{\mu}-\mathcal{K} k^{m}-\mathcal{N} k^{n}$ [5] providing the amplification rate for periodic disturbances (with wave vector $\mathbf{k}$ ) of a planar interface. The term proportional to $\lambda$ is the Kardar-Parisi-Zhang (KPZ) nonlinearity, generically expected whenever the interface evolves in the absence of conservation laws [3], while $\eta_{\mathbf{k}}(t)$ is Gaussian uncorrelated noise. Indeed, equations like (1) have been derived from constitutive laws, and from symmetry arguments, as weakly nonlinear, long wavelength $(k=|\mathbf{k}| \ll$ 1) descriptions of a variety of systems $[1,3,6]$. Locality holds whenever $\omega_{\mathbf{k}}$ is a polynomial in $k^{2}$, as for the celebrated Kuramoto-Sivashinsky (KS) equation [6] ( $\mu=$ $2, m=4)$. In contrast, nonlocality is associated with odd or, generically, noninteger powers of $k$, Eq. (1), depending on slowly decaying kernels in real space [7]. Important examples are the MS or Saffman-Taylor instabilities ( $\mu=$ $1, m=3$ ) [8] and also the Darrieus-Landau instability occurring in the propagation of a premixed laminar flame [9], for which the gas expansion produced by heat induces wrinkles on the flame front, and $\mu=1, m=2$ [10]. Remarkably, growing interfaces controlled by ballistic transport are described by an identical dispersion relation [11]. In all these cases, the instability is induced by the $-\nu k^{\mu} h_{\mathbf{k}}(t)$ term. In general, the unstable $(\nu<0)$ nonlocal Eq. (1) has been proposed as a universal description of systems in which long-range interactions persist at the level of amplitude descriptions [7], while its stable $(\nu>$ 0 ) counterpart, studied in the context of kinetic roughening for the $\lambda=0$ case [3], has been proposed [12] as a description of surface growth mediated by long-range surface hopping mechanisms like Lévy flights. Indeed, $k^{\mu} h_{\mathbf{k}}(t)$ is a fractional power of the Laplacian operator, employed to describe anomalous diffusion [13]. Moreover, unstable dispersion relations of the same form as in Eq. (1) have been seen to describe effectively (for $0<\mu<2, m=2$ ) experiments of surface growth by electrodeposition [14]. 
While the stable version of Eq. (1) leads to scale invariant interfaces whose critical exponents follow from dimensional analysis for $\mu=1$ [2], and can be obtained analytically for generic $\mu$ [15], the behavior for the unstable cases has remained poorly understood. In this work, we show that they are novel and unexpected, having a number of remarkable features. While, as in the KS system, the KPZ nonlinearity stabilizes the short time instability, now it does not control the asymptotic scaling for a whole parameter range. This is, rather, controlled by the $-\nu k^{\mu} h_{\mathbf{k}}(t)$ term, and differs from that of the stable (DLEtype) case because (i) it does not follow from dimensional analysis, (ii) it is $d$-independent, and (iii) associated with a Galilean invariance that is hidden (nonexplicit) in Eq. (1).

In order to be specific, we will consider the cases for which $m=2$, and $0<\mu \leq 2$. We have checked that the long-distance properties are unmodified for stabilizing terms with $m>2$ and for nonzero $\mathcal{N}$. Note that, while the $\mu=2$ limit of Eq. (1) (in the unstable case) is the (noisy) $\mathrm{KS}$ equation, cases with $\mu<2$ correspond to superdiffusive surface relaxation. A conspicuous instance is $\mu=1$, for which relaxation is ballistic, with Eq. (1) becoming a stochastic generalization of the MichelsonSivashinsky (SMS) equation, derived, e.g., for reactive infiltration in porous media [16]. In the deterministic case $(\eta=0)$, it was obtained [10] for premixed laminar flames near instability onset. Now $\omega_{\mathbf{k}}$ incorporates the



FIG. 1 (color online). $\quad S(k, t)$ curves for Eq. (1) $(d=1)$ with $\mu=1$ (times between $t=200$ and $t=2000$, bottom to top) (a), and $\mu=1 / 2$ (times between $t=20$ and $t=100$ ) (b). In each panel the stable case is the (single color) lower set of curves and the unstable case is the (two color) upper set. Insets provide $W(t)$ vs $t$ for the unstable (lower curve, lower right axes) and stable (upper curve, upper left axes) conditions. Parameters are $|\nu|=$ $\mathcal{K}=\lambda=1$ and $D=10^{2}$ (a) and $10^{4}$ (b) $(D=1$ for the stable conditions). System size is $L=2^{14}$, except for the un stable cases $L=2^{10}$ (a) and $2^{13} / 5$ (b). All axes are in arbitrary units. In each case $k_{\max }=\pi / \Delta x$ with $\Delta x$ the space discretiza tion unit.
Darrieus-Landau instability, with $\mathcal{K}$ being related with the Markstein length in the combustion problem [9].

We can thus gain insight into the dynamics described by Eq. (1) by first considering the $\mu=1$ SMS case. For convenience, notice that, for $\mathcal{N}=0$, Eq. (1) depends on a single independent parameter after appropriate rescaling of $h, \mathbf{k}$, and $t$; we choose it to be the noise amplitude $D$. We show in Fig. 1 the evolution of the global surface roughness $W(t)$ (root mean square fluctuation of the surface height) as computed from a numerical integration of Eq. (1) with $\mu=1$, using the pseudospectral method described in $[8,17]$. As we see [inset of Fig. 1(a)], in the unstable case an initial transient is followed by a fast increase of $W$ due to the linear instability, that is followed by power law growth of the form $W(t) \sim t^{\beta}$ with $\beta=1.14$. Qualitatively, and similarly to the KS system, nonlinear effects stabilize the morphological instability, although they do not operate in exactly the same fashion, as suggested by the noiseless limits: while nonlinear cell interaction leads to spatiotemporal chaos in the KS case $[3,6]$, the (long time) nonlinear shape reached for the MS equation is a giant cusp that responds to noisy perturbations by creation or annihilation of smaller cusps [18]. Quantitatively, the value of $\beta$ signals very fast growth in the context of surface kinetic roughening [3], but Fig. 1 proves it to be associated with genuine surface scale invariant behavior. The power spectral density or surface height structure factor $S(k, t)=\left\langle|h(k, t)|^{2}\right\rangle$ is shown in Fig. 1(a) for different times (two-color set of curves). While short times are dominated by the peak associated with the linear instability, for longer times the $S(k, t)$ curves actually fulfill the Family-Vicsek scaling ansatz [3], leading to asymptotic scaling of the form $S(k, t \rightarrow$ $\infty) \sim 1 / k^{2 \alpha+d}$ with a value of the roughness exponent that we estimate from data collapse [3] as $\alpha=$ $1.05 \pm 0.05$. Likewise we estimate a dynamic exponent value $z=0.92 \pm 0.05$. These values contrast strongly with those of the stable case [2,3], $\alpha=0$ and $z=1$, implying $\beta=\alpha / z=0$, namely, logarithmic increase of the roughness; see the upper curve in the inset of Fig. 1(a).

Numerically, for the unstable condition we obtain exponent values compatible with $\alpha+z=2$, and $z=\mu$. Moreover, these equalities hold in higher dimensions, as can be checked in [19] for the $(\mu=1)(2+1)$ dimensional case, where we obtain $\alpha=1.10 \pm 0.05, z=$ $0.90 \pm 0.05$. In general, the large value of the roughness exponent implies a strong persistence in the height fluctuations, leading to suppression of small surface features. Actually, for $\alpha=1$ the surface height field is a self-similar (rather than self-affine) fractal, related with the disordered but somehow hierarchical morphologies (small cusp motion [18]) that are produced [20]. On the other hand, $z=1$ relates with the ballistic nature of the surface relaxation mechanism implied by the $-\nu k h_{\mathbf{k}}(t)$ term in the evolution equation. Experimental realizations of these scaling relations are available, e.g., for plasma-etched $\mathrm{Si}(100)$ interfaces $\alpha=0.96$ and $z=1.05$ [21]. 
We have also studied Eq. (1) for other values of $\mu$, both for $(1+1)$ - and $(2+1)$-dimensional systems. Results are shown in Fig. 1(b) and [19] for $\mu=1 / 2<1$, that corresponds to an unexplored (to our knowledge) instance of unstable superballistic (Lévy flight type) interface relaxation [12]. As we see in the figures, the qualitative behavior is similar to the $\operatorname{SMS}(\mu=1)$ case, especially at large length scales. Quantitatively, we obtain $\alpha=1.52 \pm 0.03$, $z=0.44 \pm 0.03$ in $d=1$ and $\alpha=1.55 \pm 0.05, z=$ $0.45 \pm 0.05$ in $d=2$. As a reflection of the strong relaxation mechanism note, however, how $S(k, t)$ increasingly approaches for long times and high $k$ the power law that sets in at large distances, leading eventually to a single power law describing the whole range of scales in the system. This contrasts markedly with the time evolution of the power spectrum for local interfaces (even in the unstable case) displaying crossover phenomena [3], and even with the SMS behavior. On the other hand, the roughness and dynamic exponents change quantitatively as compared with the latter, albeit still fulfilling the $d$-independent scaling relations $z=\mu$ and $\alpha+z=2$. In our simulations this seems to be the case for all values $0<\mu<z_{\mathrm{KPZ}}(d)$, where $z_{\mathrm{KPZ}}(d)$ is the dynamic exponent of the KPZ equation for $d$-dimensional substrates $(3 / 2$ for $d=1,1.61$ for $d=2$ [22]). At each $d(=1,2)$, we obtain $\alpha$ and $z$ exponents that take ( $d$-dependent) KPZ values for $z_{\mathrm{KPZ}}(d) \leq$ $\mu \leq 2$ [23]. Note that $\mu \rightarrow 2^{-}$is not a well-defined limit for fixed $m=2, \mathcal{N}=0$. In this case, we allow, e.g., for $\mathcal{N} \neq 0$ with $n=4$ [5], the $\mu=2$ limit becoming the stochastic KS equation. In the noisy case, the scaling properties are indeed expected to be those of the KPZ equation [24,25].

Note that the exponent relation $\alpha+z=2$ is well known to be associated with the Galilean invariance of the KPZ equation $[3,26]$. In our case it comes as a surprise since power counting arguments [2,3] suggest the irrelevance of the KPZ nonlinearity. Indeed, if we rescale $\tilde{h}(\mathbf{r}, t)=$ $b^{-\alpha} h\left(b \mathbf{r}, b^{z} t\right)$ with $b>1$, then $\tilde{h}$ satisfies Eq. (1) with modified parameters $\tilde{\nu}=b^{z-\mu} \nu, \quad \tilde{\mathcal{K}}=b^{z-2} \mathcal{K}, \quad \tilde{\lambda}=$ $b^{\alpha+z-2} \lambda$, and $\tilde{D}=b^{z-2 \alpha-d} D$. Since $\nu$ is the most relevant parameter, scale invariance is expected with $z=\mu$ and $z=2 \alpha+d$. The latter relation is hyperscaling, associated with nonrenormalization of the noise amplitude, given that the $\mathcal{K}=\mathcal{N}=\lambda=0$ limit of Eq. (1) is variational and has (in the stable case) the asymptotic height distribution $\mathcal{P}\{h\} \propto \exp \left[(-\nu / 2) \int k^{\mu}\left|h_{\mathbf{k}}\right|^{2} d \mathbf{k}\right]$. Indeed, all these properties are fulfilled for $\nu>0$, e.g., the ensuing negative values of $\alpha=(\mu-d) / 2$ for small enough $\mu$ signalling flat interfaces for $d \geq 1$ (see insets of Fig. 1 and [19]) $[2,12,15]$. Moreover, these results for the morphologically stable condition suggest that Eq. (1) is not Galilean invariant [8], as confirmed through the usual tilt transformation [3].

In order to account for the scaling properties of the unstable condition we thus need to improve on the previous dimensional analysis. To this end, we perform a dynamic renormalization group (DRG) study of Eq. (1) for $m=2$ and $\mathcal{N}=0$. Following the standard procedure [26,27], we arrive at the following one loop RG parameter flow for arbitrary $d$ :

$$
\begin{gathered}
\frac{d \nu}{d l}=\nu[z-\mu], \quad \frac{d \lambda}{d l}=\lambda[\alpha+z-2], \\
\frac{d \mathcal{K}}{d l}=\mathcal{K}\left[z-2-\frac{\lambda^{2} D K_{d}}{4 d} \frac{(d-2) \mathcal{K}+(d-\mu) \nu}{\mathcal{K}(\mathcal{K}+\nu)^{3}}\right], \\
\frac{d D}{d l}=D\left[z-2 \alpha-d+\frac{\lambda^{2} D K_{d}}{4(\mathcal{K}+\nu)^{3}}\right]
\end{gathered}
$$

where the coarse graining is performed in an infinitesimal shell $k \in[\Lambda(1-d l), \Lambda]$ within the band of linearly stable (large) wave vector values, we fix the lattice cutoff, $\Lambda=1$, and $K_{d}=2 /\left[(4 \pi)^{d / 2} \Gamma(d / 2)\right]$. Naturally, Eqs. (2)-(4) generalize the KPZ flow for nonzero $\mu$ and inherit the known analytical limitations of the latter [3,27]. Still, they carry valuable information. Thus, a detailed analysis yields the same fixed point structure as for the KPZ equation, with the addition of two new nontrivial fixed points. One is associated with hyperscaling $(z=\mu, z=2 \alpha+d)$ and corresponds to the stable interfaces, while the second one implements Galilean invariance $(z=\mu, \alpha+z=2)$, and is the one found in our simulations for the morphologically unstable condition. For $d=1$ and increasing $\mu$, this fixed point merges with the KPZ one at exactly $\mu=z_{\mathrm{KPZ}}(1)$, losing stability in favor of the latter for larger values $z_{\mathrm{KPZ}}(1) \leq \mu \leq 2$, justifying our numerical observation of KPZ scaling in this range of $\mu$. For $d=2$, as it is well known, the KPZ fixed point is at infinity while the Galilean fixed point remains finite only up to $\mu=4 / 3<z_{\mathrm{KPZ}}(2)$. Our simulations indicate KPZ scaling for $z_{\mathrm{KPZ}}(2)<\mu \leq$ 2. In general for $d=1,2$, the competition between the noise and the nonlinear terms [28] induces the KPZ value for the roughness exponent which, combined with Galilean invariance, yields $\nu$ irrelevant for $\mu \geq z_{\mathrm{KPZ}}(d)$ (albeit still without $\nu$ renormalization, at variance with the KS case

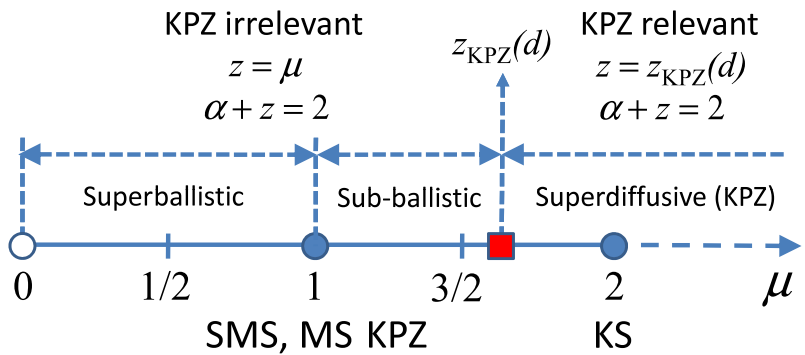

FIG. 2 (color online). Summary of scaling properties of Eq. (1) for $\mu \in(0,2]$, extrapolated to general $d$. MS KPZ has $\mu=1$, $m=3$ [8], being asymptotically equivalent to the SMS equation. $\mathrm{KS}$ denotes the noisy KS equation. $3 / 2 \leq z_{\mathrm{KPZ}}(d)$ increases for increasing $d \geq 1$ [22]. 
[3]). Given that $z_{\mathrm{KPZ}}(d) \leq 2$ all the way up to the upper critical dimension [3], we hypothesize that, for Eq. (1), KPZ scaling occurs for $z_{\mathrm{KPZ}}(d) \leq \mu \leq 2$. We summarize the asymptotic properties of the family of unstable nonlocal equations (1) for $0<\mu \leq 2$ in Fig. 2, marking cases for which simulations are provided.

Although the fixed point structure of the RG flow agrees with our numerical simulations, several questions arise. On one hand, there is the interference [3] between the typical length scale set in by the morphological instability and the analytical structure of the flow [the pole of Eqs. (3) and (4) for $\mathcal{K}+\nu=0][24,25]$. On the other hand, the role of the KPZ nonlinearity in Eq. (1) is very special. Thus, while here it does not control the scaling properties for all parameter values, in marked contrast with the KS case (at least for $d=1$ ) [3], it is definitely required as in the latter in order to stabilize the system dynamically for intermediate to long times. In the RG language, as long as $\mu<z_{\mathrm{KPZ}}(d)$, the $\mathrm{KPZ}$ nonlinearity needs to renormalize somehow to zero in infinite RG flow "time." This may be related with the peculiar fact that $\lambda$ is a singular perturbation to the linear equation in (1), and while the exponents are not those of the KPZ universality class for these $\mu$ values, they do satisfy its Galilean invariance, even though the dynamical equation does not. Notice that such a symmetry allows one, through Eq. (2), to have a nonzero $\lambda$ at the fixed point. Thus, in some way the asymptotic dynamics preserves the nonlinearity required for dynamical stability. The ensuing Galilean scaling relation, combined with the one originating in power counting (the condition for nonzero $\nu$ at the fixed point), allows our RG flow to succeed in describing the results of the numerical simulations, in a way that is reminiscent of the "accidental" success of the DRG for the $d=1 \mathrm{KPZ}$ equation [27]. In any case, one may think of the Galilean symmetry as hidden: even though the underlying equation does not have it, the hydrodynamic behavior displays it. Explicit Galilean invariance of the dynamical equation seems sufficient but not necessary for it to be a feature of the largescale properties.

Beyond their role as realistic descriptions of experimental interfaces (additional examples exist for electrodeposition [14] and for unstable flame propagation [29]), these equations are interesting for their combination of nonlocality and fluctuations, having remained poorly understood to date [7]. Moreover, they may account for similarities between diffusive and ballistic surface growth $[8,11]$. Finally, still several theoretical puzzles (as, e.g., the $d$ independence of the exponents in the small $\mu$ range) remain as a challenge for future research.

This work has been partially supported through Grants No. FIS2006-12253-C06-01 and No. FIS2006-12253-C0606 (MEC, Spain) and No. S-0505/ESP-0158 (CAM, Spain). M.N. acknowledges support by Fundación Carlos III (Spain) and by Fondazione Angelo Della Riccia (Italy).
[1] P. Pelcé, New Visions on Form and Growth (Oxford University, New York, 2004).

[2] J. Krug and P. Meakin, Phys. Rev. Lett. 66, 703 (1991).

[3] J. Krug, Adv. Phys. 46, 139 (1997).

[4] S. G. Mayr and R.S. Averback, Phys. Rev. Lett. 87, 196106 (2001).

[5] The $\mathcal{N} k^{n}$ term is a higher order correction to $\mathcal{K} k^{m}$ respecting the asymptotic behavior; it will only be con sidered when needed for technical reasons.

[6] Y. Kuramoto Chemical Oscillation, Waves and Turbulence (Springer, Heidelberg, 1984).

[7] K. Kassner and C. Misbah, Phys. Rev. E 66, 026102 (2002).

[8] M. Nicoli, M. Castro, and R. Cuerno, Phys. Rev. E 78, 021601 (2008).

[9] V. V. Bychkov and M. A. Liberman, Phys. Rep. 325, 115 (2000).

[10] G. I. Sivashinsky, Acta Astronaut. 4, 1177 (1977); D. M. Michelson and G. I. Sivashinsky, ibid. 4, 1207 (1977).

[11] G. S. Bales et al., Science 249, 264 (1990).

[12] J.A. Mann, Jr. and W. A. Woyczynski, Physica (Amsterdam) 291A, 159 (2001).

[13] R. Metzler and J. Klafter, Phys. Rep. 339, 1 (2000).

[14] M. Nicoli, M. Castro, and R. Cuerno, J. Stat. Mech. (2009) P02036.

[15] P. Biler, G. Karch, and W. A. Woyczynski, Stud. Math. 135, 231 (1999).

[16] P. Kechagia, Y.C. Yortsos, and P. Lichtner, Phys. Rev. E 64, 016315 (2001).

[17] R. Gallego, M. Castro, and J. M. López, Phys. Rev. E 76, 051121 (2007).

[18] See V. Karlin, Math. Models Methods Appl. Sci. 14, 1191 (2004), and references therein.

[19] See EPAPS Document No. E PRLTAO 103008929 for the analog of Fig. 1 in $d=2$. For more information on EPAPS, see http://www.aip.org/pubservs/epaps.html.

[20] See EPAPS Document No. E PRLTAO 103008929 for surface evolution movies. For more information on EPAPS, see http://www.aip.org/pubservs/epaps.html.

[21] Y. P. Zhao et al., Phys. Rev. Lett. 82, 4882 (1999).

[22] E. Marinari, A. Pagnani, and G. Parisi, J. Phys. A 33, 8181 (2000).

[23] See EPAPS Document No. E PRLTAO 103008929 for scaling behavior when $z_{\mathrm{KPZ}}(d) \leq \mu \leq 2$ for $d=1$, 2. For more information on EPAPS, see http://www.aip.org/ pubservs/epaps.html.

[24] R. Cuerno and K. B. Lauritsen, Phys. Rev. E 52, 4853 (1995).

[25] K. Ueno, H. Sakaguchi, and M. Okamura, Phys. Rev. E 71, 046138 (2005).

[26] W. D. McComb, The Physics of Fluid Turbulence (Oxford University, New York, 1991).

[27] T. Halpin Healy and Y. C. Zhang, Phys. Rep. 254, 215 (1995).

[28] H. G. E. Hentschel and F. Family, Phys. Rev. Lett. 66, 1982 (1991).

[29] G. Searby, J. M. Truffaut, and G. Joulin, Phys. Fluids 13, 3270 (2001). 